\title{
White matter microstructural differences underlying beta oscillations during speech in adults who stutter
}

Article

Accepted Version

Creative Commons: Attribution-Noncommercial-No Derivative Works 4.0

Mollaei, F. ORCID: https://orcid.org/0000-0003-2916-9750, Mersov, A., Woodbury, M., Jobst, C., Cheyne, D. and De Nil, L. (2021) White matter microstructural differences underlying beta oscillations during speech in adults who stutter. Brain and Language, 215. 104921. ISSN 0093-934x doi: https://doi.org/10.1016/j.bandl.2021.104921 Available at https://centaur.reading.ac.uk/97892/

It is advisable to refer to the publisher's version if you intend to cite from the work. See Guidance on citing.

To link to this article DOI: http://dx.doi.org/10.1016/j.bandl.2021.104921

Publisher: Elsevier

All outputs in CentAUR are protected by Intellectual Property Rights law, including copyright law. Copyright and IPR is retained by the creators or other copyright holders. Terms and conditions for use of this material are defined in the End User Agreement. 


\section{CentAUR}

Central Archive at the University of Reading

Reading's research outputs online 
Title: White matter microstructural differences underlying beta oscillations during speech in adults who stutter

\section{Authors:}

Fatemeh Mollaei, Ph.D.*,a,b, Anna Mersov, M.Sc. ${ }^{a}$, Merron Woodbury, B.Sc. ${ }^{\text {b }}$, Cecilia Jobst, M.Sc. ${ }^{b}$, Douglas Cheyne, Ph.D. ${ }^{\mathrm{a}, \mathrm{b}, \mathrm{d}, \mathrm{e}}$, Luc De Nil, Ph.D. ${ }^{\mathrm{a}, \mathrm{c}}$

${ }^{a}$ Department of Speech-Language Pathology, University of Toronto, 500 University Street, Toronto, Ontario M5G 1V7, Canada

${ }^{b}$ Program in Neurosciences and Mental Health, The Hospital for Sick Children Research Institute, Toronto, Ontario M5G 0A4, Canada

${ }^{\mathrm{c}}$ Rehabilitation Sciences Institute, Toronto, Ontario M5G 1V7, Canada

${ }^{\mathrm{d}}$ Institute of Medical Sciences and Institute of Biomaterials and Biomedical Engineering, University of Toronto, Toronto, Ontario M5S 2J7, Canada

${ }^{\mathrm{e}}$ Department of Medical Imaging, University of Toronto, Toronto, Ontario M5T 1W7, Canada

\section{*Corresponding author:}

Fatemeh Mollaei, Ph.D.

Department of Speech-Language Pathology

Rehabilitation Sciences Building

University of Toronto

160-500 University Avenue

Toronto, Ontario M5G 1V7, Canada

Email: Fatemeh.mollaei@utoronto.ca 


\begin{abstract}
The basal ganglia-thalamocortical (BGTC) loop may underlie speech deficits in developmental stuttering. In this study, we investigated the relationship between abnormal cortical neural oscillations and structural integrity alterations in adults who stutter (AWS) using a novel magnetoencephalography (MEG) guided tractography approach. Beta oscillations were analyzed using sensorimotor speech MEG, and white matter pathways were examined using tract-based spatial statistics (TBSS) and probabilistic tractography in 11 AWS and 11 fluent speakers. TBSS analysis revealed overlap between cortical regions of increased beta suppression localized to the mouth motor area and a reduced fractional anisotropy (FA) in the AWS group. MEG-guided tractography showed reduced FA within the BGTC loop from left putamen to subject-specific MEG peak. This is the first study to provide evidence that structural abnormalities may underlie functional deficits in stuttering and reflect a network deficit within the BGTC loop that includes areas of the left ventral premotor cortex and putamen.
\end{abstract}

Key Words: developmental stuttering, magnetoencephalography, diffusion tensor imaging, white matter, probabilistic tractography, speech production 


\section{Introduction}

Speech production is a highly complex sensorimotor process that requires coordination of articulatory movements in an internally timed manner. Different theoretical models of speech production have been proposed in an attempt to outline the neural network and temporal dynamics of speech production. For instance, the Directions into Velocities of Articulators (DIVA; Guenther, 2016) model and the State Feedback Control (SFC) model of speech (Houde \& Nagarajan, 2011) both specify the role of feedforward and feedback control processes in speech and their associated neural processes. The basal ganglia-thalamocortical loop (BGTC) has been proposed to be involved in the generation of motor commands within the feedforward control system in an internally timed manner. A breakdown in the timing of these commands has been proposed to give rise to stuttering behaviour (Alm, 2004; Chang \& Guenther, 2020; Etchell et al., 2014). According to these models, if the commands to initiate or terminate components of speech output are affected, blocking or repetitions of sounds and syllables may be observed.

Developmental stuttering affects approximately $1 \%$ of the adult population (Reilly et al., 2009) and thought to be a manifestation of impaired speech planning and execution that disrupts the forward flow of speech. It has its onset during childhood and, unless early spontaneous recovery occurs, persists into adulthood. Salient symptoms of stuttering include sounds or syllable repetitions, as well as sound prolongations and pauses (WHO, 2016). At the neural level, multiple studies using neuroimaging techniques such as functional and structural MRI have reported differences in the speech network in adults and children who stutter compared to fluent speakers (for a review see Chang \& Guenther, 2020). Several studies have reported white matter differences in the left Rolandic operculum region underneath the sensorimotor representation of the oropharynx at the level of the central sulcus in adults who stutter (AWS) compared to fluent 
speakers (Cai et al., 2014; Sommer et al., 2002; Watkins et al., 2008). Diffusion MRI studies using voxel-wise analysis such as Tract Based Spatial Statistics (TBSS; Smith et al., 2006) have reported reduced fractional anisotropy (FA) in AWS compared to fluent speakers in the left posterior inferior frontal gyrus (pIFG), ventral and premotor cortex, and middle primary motor cortex (Cai et al., 2014; Chang et al., 2008; Connally et al., 2014; Sommer et al., 2002). FA quantifies the degree of directionality (i.e., anisotropy) of water diffusion in the tissue (Basser et al., 1994). It has been found that reduced FA is related to anomalies in white matter structure such as axonal density and degree of myelination (Beaulieu, 2014). Using probabilistic tractography that measures region to region white matter connectivity, Chang and Zhu (2013) found reduced connectivity in the BGTC loop in children who stutter (CWS), specifically between the putamen and inferior frontal cortex pars opercularis (BA 44) and the supplementary motor area (SMA) in the left hemisphere, and between the inferior frontal gyrus and putamen, bilaterally. These findings were corroborated using resting-state functional connectivity. In addition, using MRI and structural equation modelling, Lu et al. (2010) found stronger connectivity from the putamen to the thalamus, as well as, from the thalamus to the temporal and pre-SMA regions in the left hemisphere in AWS. Simultaneously, they found weaker connectivity from the posterior middle temporal gyrus to the putamen. Along with these functional connectivity deficits, they also reported more gray matter volume concentration in the left putamen, less gray matter volume concentration in the left medial frontal gyrus and anterior superior temporal gyrus, and less white matter volume concentration underlying the left posterior superior temporal gyrus inside the BGTC loop (Lu et al., 2010). In addition, Sowman et al. (2017) reported lower gray matter volume concentration in the left caudate of the basal ganglia in AWS. Relatedly, in another study using functional MRI, stuttering severity was found to be 
positively correlated with the caudate activity bilaterally prior to speech therapy (Giraud et al., 2008). However, Toyomura et al. (2011) observed a negative correlation between the putamen and stuttering severity, bilaterally. They also found reduced activity within the basal ganglia during normal reading using functional MRI. This reduction was normalized during fluencyinduced conditions (i.e., chorus and metronome-timed). The findings above therefore point to an impairment in BGTC loop both functionally and structurally in AWS.

While structural and functional MRI findings provide valuable information regarding the neural location and connectivity patterns of speech deficits within the BGTC circuit in AWS, they lack temporal precision at the neural level. Speech production relies heavily on precisely timed signals and mapping the temporal aspects of speech movement to neural activity is necessary for understanding the neurophysiology of stuttering. The recording of magnetoencephalography (MEG) and electroencephalography (EEG) allows investigation of the temporal dynamic of neural communication (oscillations) during the planning and production of speech (De Nil et al., submitted; Jenson et al., 2014; Mersov et al., 2016). Neural oscillations have been found to facilitate communications between different neural structures and subsystems (Fries, 2005). Oscillatory power within specific frequency bands provides multiple information regarding motor and sensory systems. As pertinent to speech production, beta oscillations in the $13-30 \mathrm{~Hz}$ range have been hypothesized to have a role in functional coordination between auditory and motor systems (Fujioka et al., 2012; Gehrig et al., 2012; Jenson et al., 2014; Salmelin \& Sams, 2002). Activity within the beta band is thought to encode motor information within premotor and primary motor cortices (Pfurtscheller, 1981; Schnitzler et al., 2000). In addition, beta activity has been found to be involved in cuing the initiation and termination of internally-stored motor programs (Bartolo \& Merchant, 2015; Etchell et al., 2015). Bartolo and 
Merchant (2015) found increased beta activity in the putamen of the basal ganglia in monkeys at the beginning of movement sequences and suggested that basal ganglia produce a general initiation signal that engages the motor system in different sequential motor tasks. Beta activity prior to speech production has also been reported to be involved in the generation of motor programs through feedforward control as well as in sending the motor programs to the cortical motor, premotor, and sensory regions for execution and sensory monitoring (Hickok, 2012; Kilavik et al., 2013; Liljeström et al., 2015; Piai et al., 2015). For example, Liljeström et al. (2015) found enhanced beta band coherence within a network involving the bilateral primary, premotor, and auditory cortices preceding speech production. Based on the reports above, it appears that beta oscillations may have two specific roles during speech production: one in the feedforward control system through the initiation and termination of motor programs within the BGTC circuit, and the other in the feedback control system through the interaction of motor programs with the sensory cortices for error monitoring and updating of motor programs.

In developmental stuttering, multiple studies have reported impaired beta activity during preparation and execution of speech (Kikuchi et al., 2017; Mersov et al., 2016, 2018; Saltuklaroglu et al., 2017; Sengupta et al., 2016). In a recent MEG study, Mersov et al. (2016) reported increased beta suppression during the preparation and execution of speech at the left ventral premotor cortex (mouth motor area, BA 6) in AWS compared to fluent speakers. They argued that beta suppression during speech preparation suggests a reduced coordination and automaticity in the speech motor network. This is consistent with the suggested role of beta oscillations in the feedforward control of speech, also enabling proper monitoring of the sensory consequences of speech during feedback control (Gehring et al., 2012; Guenther \& Chang, 2020; Hickok, 2012). However, using EEG and time-frequency analysis, Jenson et al. (2014) reported 
reduced mu-beta suppression in AWS compared to fluent speakers (FS) during speech production. Reviewing the literature, Jenson et al. (2020) interpreted their previous findings of reduced mu-beta desynchronization in AWS as evidence of a weakened feedforward control of speech (Max et al., 2004). Some of the differences between various studies may be related to methodological differences, including the use of MEG vs. EEG, as well as differences in speech stimuli selection and presentation. Regardless of any differences, an outstanding question remains as to the neural source of the observed functional beta differences between AWS and typically fluent speakers.

In the current study, we aimed to integrate our previously reported functional MEG results (Mersov et al., 2016) with structural white matter differences as measured by diffusion tensor imaging (DTI) in order to assess the integrity of the BGTC loop in the same group of AWS and fluent speakers in order to uncover the underlying source of the differences in beta oscillations observed between stuttering and nonstuttering speakers. All MEG and DTI data were collected as part of a larger research project (Mersov et al., 2016). In this paper, we report the results of the DTI analysis to the already reported MEG results elsewhere (Mersov et al., 2016). Specifically, we used the MEG-localized activity in the speech-motor areas to guide TBSS and probabilistic tractography in order to investigate the possible relationship between beta oscillatory responses and any underlying structural deficits within the BGTC loop. Our main hypothesis was that feedforward deficits are related to abnormal functional beta oscillations that occur as a result of an impaired BGTC loop. To test our hypothesis, we compared bilateral cortical regions where there was an overlap of functional and structural impairment using voxelwise analysis of TBSS and MEG-guided probabilistic tractography. With the TBSS analysis, we aimed to highlight region-specific FA and MD white matter differences that correlate with the 
left-lateralized beta suppression previously found in AWS (Mersov et al., 2016). Adding a MEGguided tractography approach, we aimed to investigate the presence of a direct association between functional beta oscillations and measures of region to region structural connectivity patterns within the BGTC loop in AWS. To our knowledge, this is the first study to combine MEG and DTI recordings in the same group of participants and examine the role of brain function and structure within BGTC loop in developmentally stuttering adults.

\section{Materials and Methods}

\subsection{Participants}

11 AWS (mean age 30 years, range 21-45, 2 females) and 11 age- and gender-matched control group of fluent speakers (FS, mean age 32, range 21-43, 2 females) were included in the current study. The participants were tested as part of a study reported by Mersov et al. (2016). However, in that paper data were reported for a total of 24 participant (12 participants in each group). For the analysis reported in this paper, we excluded two participants, one from the control group and one from the AWS group, because DTI data were not available for those participants. The institutional review board of the Hospital for Sick Children (SickKids) and University of Toronto reviewed and approved this study. Informed consent was obtained from each participant prior to the study. All participants were right-handed based on the Edinburgh Handedness Inventory (Oldfield, 1971). None self-reported neurological conditions that may have affected speech, vision, motor ability, or hearing. English was their primary language of use. In addition, the control participants reported no history of speech and language impairments or speech therapy. All AWS self-reported that they have had different types of interventions from fluency shaping to stuttering modification since stuttering onset, and that they have been stuttering early childhood. However, none of the participants in the AWS group were enrolled in 
stuttering therapy at the time of testing. In order to be included in the AWS group, participants scored at least "very mild" in the Stuttering Severity Index (SSI-4; Riley, 1972), as assessed by a qualified speech-language pathologist (See Table 1 for SSI-4 scores). The AWS participants were recruited from the Speech and Stuttering Institute, Toronto, Canada, and the control participants were recruited by word of mouth and through recruitment flyers posted on campus and around the city.

\subsection{MEG task and data acquisition}

The data were collected as part of a larger research project that included additional experiments for which the results are reported elsewhere (Mersov et al., 2016, 2018). Briefly, participant-specific word stimuli were presented in random order, and participants were instructed to say each word as the initial word in a carrier phrase (" $[\mathrm{X}]$ is a word" with $[\mathrm{X}]$ indicating a participant-specific target word). A more detailed description of how the word stimuli were selected can be found in Mersov et al. (2016). Participants were asked to complete the entire phrase, even if they stuttered on the utterance or were delayed in another way. Each trial started with a target fixation cross in the middle of the screen that was presented for a randomly alternating duration of 1 or $2 \mathrm{~s}$. The stimulus "[X] is a word" then appeared for 500 ms, followed by a $500 \mathrm{~ms}$ blank screen. A cue immediately followed in the form of " $<$ )))" and remained for $3 \mathrm{~s}$. This cue prompted participants to speak the stimulus phrase out loud. The cue was followed by a fixation cross that initiated the next trial. During a practice session, participants were acquainted with the task using a sequence of eight test words prior to taking their seat in the MEG. The experiment ran in 4 blocks, each with 55 trials. A 5 s message was displayed between blocks to notify the participant of their progress. The task was performed in a seated position. 
Neuromagnetic activity was recorded continuously using a 151-channel whole head CTF MEG system located at the Hospital for Sick Children in Toronto, Canada. All data signals were collected at 12,0000 samples/s and band passed at $0-4000 \mathrm{~Hz}$ to preserve acoustic integrity of the speech signal. MEG data were filtered off-line to $0.4-250 \mathrm{~Hz}$ and down-sampled to 1000 samples/s. Verbal responses were recorded using a Rode NTG-2 directional condenser microphone placed about $1.8 \mathrm{~m}$ from the participant and recorded as a secondary channel.

\subsection{MRI/DTI acquisition}

Immediately following the MEG session, neuroanatomical images were acquired from all participants using a 3-T Siemens Trio MRI scanner (Siemens Healthcare, Germany). Diffusionweighted data was collected via echo planar imaging (EPI) sequence, each with the same 21 noncollinear directions $(\mathrm{TE}=87 \mathrm{~ms}, \mathrm{TR}=8800 \mathrm{~ms}$, matrix size: $122 \times 122$, FOV: $21.96 \mathrm{~cm}, \mathrm{~b}=$ $1000 \mathrm{~s} / \mathrm{mm}^{2}$ ) and $3 \mathrm{~b}=0$ images. In addition, T1-weighted structural images were also collected using a 3D MPRAGE gradient echo sequence (flip angle $=9^{\circ}, \mathrm{TE}=2.96 \mathrm{~ms}, \mathrm{TR}=2300 \mathrm{~ms}$ ) with 192 1-mm-thick sagittal slices (matrix size: $256 \times 256$, FOV: $25.6 \mathrm{~cm}$ ) to be utilized for DTI co-registration and MEG source localization.

\subsection{MEG-defined regions of interest}

For every participant and each hemisphere, the Synthetic Aperture Magnetometry (SAM) algorithm (Robinson \& Vrba, 1999) implemented in the BrainWave Matlab toolbox (Jobst et al., 2018; cheynelab.utoronto.ca/brainwave) was used to define a region of interest (ROI) at the maxima (peaks) of the MEG localized changes in beta band oscillations (15-25 Hz based on Mersov et al., 2016) during speech production. SAM images of induced power changes over time were generated by subtracting baseline source power activity from a sliding active time 
window of $200 \mathrm{~ms}$ duration defined at $50 \mathrm{~ms}$ intervals (starting from $1200 \mathrm{~ms}$ prior to EMG onset to $200 \mathrm{~ms}$ post EMG onset). A fixed $200 \mathrm{~ms}$ window during the fixation period ( -500 to -300 ms preceding stimulus onset) was used as baseline. Pseudo-T images were computed for beta $(15-25 \mathrm{~Hz})$ frequency bands over the entire brain at $4 \mathrm{~mm}$ resolution (for more details see: Mersov et al., 2016). The ROIs were defined at the cortex and localized to the left and right mouth motor area based on previously reported MEG analysis (BA 6; Mersov et al., 2016) on the structural T1 images and then transferred to MNI standard space. Each MEG-defined ROI consisted of 27 voxels (forming a $3 \times 3 \times 3$ voxel cube) surrounding the voxel with the global maxima. These regions of interests served as the seed and target masks for the probabilistic tractography analysis (see below).

In addition, we created contrast images of AWS minus FS for the beta power range where we found the highest peak during speech production and overlaid the contrast image on the structural TBSS results. This allowed to investigate functional-structural overlap at the level of the mouth motor area where high beta band suppression in AWS compared to FS was previously found. For this analysis, source images created in BrainWave from Mersov et al. (2016) were normalized to the MNI brain template in Analysis of Functional Neuroimages (AFNI; Cox, 1996; http://afni.nimh.nih.gov). Normalized SAM images of 15-25 Hz beta power (pseudo-T values) were analyzed statistically using t-tests with 2 groups (AWS vs. FS). We chose a onetime window of interest (600 ms to $900 \mathrm{~ms}$ ) based on the highest peak activity in Mersov et al. (2016). A cluster alpha of 0.05 was set as the threshold for statistical significance. A cluster size criterion was determined by Monte Carlo simulations using the AFNI program 3dClustSim. Monte Carlo simulations were iterated 10,000 times, with the voxel threshold being set at $p<$ 
0.01 and alpha level of 0.05 . A minimum of 6 continuous voxels in bilateral frontal cortex were required to be significant.

\subsection{Tract-Based Spatial Statistics (TBSS)}

Subject raw images were processed using the FMRIB's diffusion toolbox (FDT, FMRIB Software Library (FSL); Smith et al., 2004). After eddy current distortion and motion correction, the diffusion parameters, including fractional anisotropy, were calculated with the 'DTIFIT' software (included in the toolbox) in order to locally fit the diffusion tensors at each voxel. Voxel-wise statistical analysis of the FA data was performed using Tract-Based Spatial Statistics (TBSS; Smith et al., 2006). FA is defined as the normalized standard deviation of the magnitudes of the tensor's three eigenvectors (Basser et al., 1994). FA describes the asymmetry of diffusion along the orthogonal directions, therefore providing a measure of the degree of directionality, or anisotropy, of diffusion in the underlying tissue. FA values range from 0 to 1 , where 0 indicates the tensor is a perfect sphere (isotropic diffusion) and 1 indicates an infinitely elongated, cigarshaped ellipsoid (anisotropic diffusion, Kronfeld-Duenias et al., 2018). TBSS registers the diffusion images non-linearly to a lab-based custom-created target image obtained from a highresolution average of 58 healthy participants aligned to standard MNI space. The average of all data was reduced to a 1-mm thick white matter skeleton that aligns with the centre of white matter tracts common across participants. For each participant, the highest value of FA nearest to the skeleton was projected onto the skeleton for analysis. We used an FA threshold of 0.2 to exclude gray matter voxels. A t-test was performed at each voxel in the skeleton. The Randomise function in FSL iterated 10,0000 permutations and statistical analysis was based on an uncorrected voxel-based threshold of $p<0.005$ and cluster size threshold of 10 voxels. No cluster of voxels survived corrections for multiple comparisons using threshold-free cluster 
enhancement (TFCE; Smith \& Nichols, 2009). This is consistent with the TBSS findings from prior studies (Cai et al., 2014; Chang et al., 2008, 2015; Connally et al., 2104; Watkins et al., 2008). FA was compared between the two groups (AWS vs. FS). In addition, the group differences were further explored using Mean Diffusivity (MD) values using TBSS. MD measures how large the tensor is and provides an estimation of the overall tissue density (Pierpaoli \& Basser, 1996). Group differences for MD is reported at $p<0.005$ uncorrected.

\subsection{Probabilistic tractography analysis}

Bayesian Estimation of Diffusion Parameters Obtained using Sampling Techniques (BEDPOSTX) function was applied to estimate fiber orientation in each voxel. The PROBTRACKX2 (Behrens et al., 2003, 2007) command of FDT was then used to calculate the connectivity of seed and target regions with the following parameters: step length $=0.5 \mathrm{~mm}$; curvature threshold $=0.2$; number of samples $=5000$; number of steps per sample $=2000$; loop check $=$ True; distance correction $=$ True. In this study, we examined the connectivity patterns within the BGTC loop. The regions of putamen and thalamus were extracted based on automatically generated parcellation maps from FreeSurfer (Fischl et al., 2002). Then, we manually edited each of these regions within thalamus and putamen in each subject to be localized to the mouth motor area (Nambu, 2011). For the putamen, the mask was localized to the lower medial portion and for the thalamus, it was localized to the ventral lateral nucleus of the thalamus. This is shown on subjects' FA maps on the Figure 2 (b). We registered all of the masks including MEG-defined cortical masks to the subjects' structural space and then converted them to diffusion space to run FDT's probabilistic tractography using a custom-written Matlab toolbox. In each hemisphere, we tracked the connectivity pattern from the putamen (seed mask) to the MEG-defined cortical ROI (target mask) and vice versa (two directions) through the 
thalamus (waypoint mask). Each tract was normalized (intensity divided by waytotal, which is the number of valid generated tracts within the seed region that reach the target regions) and a probability threshold of $1 \%$ was set to reject unlikely fibers. Then, the tracts were binarized and multiplied with the individual FA, MD maps to extract the FA, MD values within the BGTC loop for each participant in each group. In addition, while the main focus of our analysis was on FA and MD, we also extracted radial and axial diffusivity (RD and $\mathrm{AD}$ ) measures in order to provide additional measures of diffusion in the main and perpendicular direction of the white matter pathways.

\subsection{Statistical Analysis}

Data were compared using analysis of variance (ANOVA), with a between-subjects factor of group (AWS vs. FS) and within-subjects factors of hemisphere (left vs. right) and connectivity directions (2 directions) for $\mathrm{FA}, \mathrm{MD}, \mathrm{RD}$, and $\mathrm{AD}$ parameters. Factor and simple effect sizes were quantified using partial $\eta_{\mathrm{p}}{ }^{2}$ (Witte \& Witte, 2010) and Cohen's $d$ (Cohen, 1992). Post-hoc testing with Bonferroni-adjusted alpha rate was used when necessary. For all the ANOVA tests, normality assumption was tested using Shapiro-Wilk tests, sphericity assumption with the Mauchly test, and the equality of variances with the Levene test. The level of significance was set at $p<0.05$ unless otherwise stated.

\section{Results}

We investigated TBSS and probabilistic tractography connectivity in the BGTC loop by combining with it previously measured MEG functional beta activity in order to determine the local and network properties of white matter structural connectivity underlying plastic changes in AWS. Below, we first describe the findings from the TBSS analysis, and its relations to the beta 
suppression during speech production. Next, we present results from our MEG-guided probabilistic tractography providing insight into the functional-structural integrity and connectivity patterns within the BGTC loop.

\subsection{TBSS results}

We performed TBSS on the diffusion data (FA and MD) from 11 AWS and $11 \mathrm{FS}$ control participants. For FA, six clusters showed significant differences at the threshold of voxel-wise uncorrected $p<0.005$ and cluster size of 10 voxels consistent with the threshold used in prior studies of TBSS in stuttering (Cai et al., 2014; Chang et al., 2008; Connally et al., 2014; Watkins et al., 2008). All six clusters showed mean FA values that were lower in the AWS group compared to the FS control group (See Table 2, Supplementary Figure 1). AWS had significantly lower FA than the FS in the following areas: (1) left superior longitudinal fasciculus underlying ventral premotor cortex; (2) left posterior corona radiata; (3) white matter underlying left inferior division of lateral occipital cortex; (4) right anterior corona radiata; (5) right superior longitudinal fasciculus underlying posterior division of supramarginal gyrus; (6) right cingulum underlying posterior division of cingular gyrus. Our data revealed functional and structural overlap with beta suppression in and around the left ventral premotor cortex (mouth motor area, BA 6) being accompanied by lower FA in the AWS group (cluster 1, Figure 1).

In addition, we examined MD to better understand the microstructure underlying functional differences in AWS vs. FS control group. For MD, four clusters showed significant differences at the threshold of voxel-wise uncorrected $p<0.005$ and cluster size of 10 voxels. All four clusters showed mean MD values higher in the AWS group compared to the FS control group (See Table 3, Supplementary Figure 2). MD was significantly higher in AWS than FS in the following clusters: (1) left white matter underlying precuneus and posterior cingulate cortex; 
(2) right superior longitudinal fasciculus underlying insular cortex; (3) right posterior thalamic radiations; (4) right white matter underlying posterior division of supramarginal gyrus. We did not observe any functional-structural overlap between MD values and MEG beta suppression in the left or right hemispheres.

\subsection{Probabilistic tractography results for BGTC loop}

Figure 2 (a) presents the MEG-defined ROIs (ventral premotor cortex, BA 6) and, Figure 2 (b) shows anatomically-defined ROIs (lower medial putamen and thalamus) overlaid on a representation of the FA maps in the MNI standard space for a participant with developmental stuttering. Figure 2 (c) also shows the binary images of the thresholded probabilistic maps of BGTC loop for the FS control group and AWS group. The mean FA and MD were extracted for both hemispheres in the two groups in two directions: (1) putamen (seed mask) to MEG-defined cortical ROI (target mask); (2) MEG-defined cortical ROI (seed mask) to the putamen (target mask). Thalamus was used as waypoint mask. The mean FA and MD values for the two directions in the AWS and FS control group are shown in Figure 3.

For FA, we observed a significant main effect of group $\left[F(1,20)=4.00 ; p=0.049 ; \eta_{\mathrm{p}}^{2}=\right.$ 0.166] with the AWS showing an increased FA, a significant main effect of hemisphere $[F(1,20)$ $\left.=12.26 ; p=0.002 ; \eta_{\mathrm{p}}{ }^{2}=0.380\right]$ with the right hemisphere showing an increased $\mathrm{FA}$, and a significant main effect of connectivity direction $\left[F(1,20)=28.37 ; p<0.01 ; \eta_{\mathrm{p}}{ }^{2}=0.586\right]$. The group by hemisphere interaction $[F(1,20)=0.970 ; p=0.337]$ and the group by connectivity direction $[F(1,20)=0.400 ; p=0.534]$ were not significant. However, the hemisphere by direction interaction was significant $\left[F(1,20)=5.718 ; p=0.027 ; \eta_{\mathrm{p}}{ }^{2}=0.222\right]$. Post hoc analysis showed significant difference for the mean FA between the right and left hemispheres with left hemisphere showing a reduced FA compared to the right hemisphere for the first direction $[t(10)$ 
$=-4.514 ; p=0.001 ;$ Cohen's $d=1.815]$ but not for the second direction $[t(10)=-0.873 ; p=$ 0.403] of AWS group (Figure 3).

For MD, there was no main effect of group $[F(1,20)=0.200 ; p=0.660]$ or direction $[F(1,20)=0.003 ; p=0.957]$. However, we observed a significant main effect of hemisphere $\left[F(1,20)=49.246 ; p<0.01 ; \eta_{\mathrm{p}}{ }^{2}=0.711\right]$ with the left hemisphere showing an increased MD compared to the right hemisphere. The group by hemisphere interaction $[F(1,20)=0.115 ; p=$ $0.738]$, the group by connectivity direction $[F(1,20)=0.663 ; p=0.425]$, and the hemisphere by direction interaction $[F(1,20)=0.068 ; p=0.797]$ were not significant. Post hoc analysis showed significant difference for the mean MD between the right and left hemispheres in the AWS group, with left hemisphere showing an increased MD compared to the right hemisphere for the first direction $[t(10)=4.631 ; p=0.001$; Cohen's $d=1.968]$ and the second direction $[t(10)=$ $3.417 ; p=0.007$; Cohen's $d=1.106]$ (Figure 3).

The analysis of RD did not yield any significant main effects for group $[F(1,20)=0.167$; $p=0.649]$, direction $[F(1,20)=0.883 ; p=0.387]$ or hemisphere $[F(1,20)=1.405 ; p=0.175]$. Similarly, none of the differences in $\mathrm{AD}$ were statistically significant: group $[F(1,20)=0.305 ; p$ $=0.763]$, direction $[F(1,20)=0.724 ; p=0.477]$ and hemisphere $[F(1,20)=1.554 ; p=0.135]$.

\section{Discussion}

This study is the first to examine structural changes that may relate to neural oscillations in stuttering. We used an established structural analysis approach (TBSS) and found overlap of beta suppression with reduced FA in the left ventral premotor cortex. This area has been reported extensively in prior TBSS studies with lower FA values in AWS (Cai et el., 2014; Chang et al., 2010; Connally et al., 2014; Sommer et al., 2002; Watkins et al., 2008). However, we did not 
find any overlap of beta suppression with other significant clusters from our TBSS findings. In addition, we employed a novel analysis approach to examine differences in the region to region connectivity of FA, MD, RD, and AD using probabilistic tractography within the basal gangliathalamocortical circuit that was guided by MEG localized beta oscillations during speech. Our main findings were reduced FA in the left hemisphere compared to the right hemisphere that was observed in the direction of basal ganglia to the mouth motor area (MEG target mask), and increased MD in the left hemisphere compared to the right hemisphere.

\subsection{Relationship between TBSS and beta oscillations findings}

The main finding of lower than normal FA in AWS compared to FS points to a white matter integrity disruption underlying left ventral premotor cortex for speech production (mouth motor area). This could point to integrity and myelination deficits of tracts underlying this area including but not limited to superior longitudinal fasciculus. The superior longitudinal fasciculus connects many speech areas, such as the inferior parietal lobe with the ventral premotor and prefrontal cortex (Catani et al., 2002), and has a role in the integration of auditory information with the motor output for fluent speech production (Chang et al., 2015; Connally et al., 2014; Rech et al., 2020). The ventral premotor cortex also contains tracts that connect the inferior frontal gyrus (IFG) to the ventral premotor cortex, forming a part of the BGTC loop, which carries the primary motor representations for articulatory commands in the ventral premotor cortex (Hickok, 2012; Chang \& Guenther, 2020). The overlap of the FA reduction in the same brain region that demonstrates beta suppression during speech is interesting, as this beta suppression was found to be increased during the preparation and execution of speech production in AWS in comparison to controls (Mersov et al., 2016). This ventral speech motor cortex likely

plays an important role in the formulation of the motor programs through the feedforward control 
within the BGTC loop (Jenson et al., 2020; Mersov et al., 2016). It could be that the FA

reduction in this area along with the abnormal functional neural oscillations give rise to abnormal initiation and timing that may cause stuttering. These initiation and timing deficits consequently may affect the updating of the motor programs through the BGTC loop and may result in the formation of less stable and less automatic motor programs in stuttering individuals. It could also be that the atypical neural processes may not only be specific to this region but be a part of a network deficit within the speech sensorimotor network. In order to answer this question, we included region to region connectivity analysis to characterize the speech network deficits of stuttering. The discussion of this analysis is outlined in the next section.

It is important to point out that although increased beta suppression found during execution of speech in AWS compared to FS was significant in the left mouth motor area (BA 6), there was also a trend for the increased beta suppression in the right mouth motor area which did not survive correction for multiple comparisons (Mersov et al., 2016). At the structural level, we did not find any differences in FA or MD between the two groups in the right mouth motor area. This result may point to the bilateral but predominantly left-lateralized contribution of sensorimotor control for the feedforward control of speech (Guenther, 2016; Hickok \& Poeppel, 2007). It has been hypothesized that the auditory feedback control of speech is mostly rightlateralized (Tourville \& Guenther, 2011) and AWS rely heavily on sensory feedback (Max et al., 2004). It could be that the structural and functional contributors of the sensory feedback control of speech are affected in the right hemisphere in developmental stuttering. These include auditory and somatosensory areas and the functional spectral parameters typically captured in the alpha band oscillations $(8-13 \mathrm{~Hz})$, which are known to be associated with somatosensory and sensorimotor feedback (Hari et al., 1997; Jenson et al., 2018). While Mersov et al. (2016) did not 
observe significant differences in the alpha band between the AWS and FS groups, they did report on a trend towards significance in the right temporal gyrus (BA 41) during speech production. In addition, they reported a positive correlation between alpha suppression during the speech preparation stage and stuttering severity in the left temporal gyrus (BA 41). Jenson et al. (2014) reported weaker mu-alpha suppression in the left hemisphere but they did not find any group differences in the right hemisphere.

This finding is also consistent with two of the theoretical models of speech production, namely the DIVA and SFC models. The DIVA model includes the BGTC loop as part of the sensorimotor network that is disrupted in stuttering. Specifically, the model proposes that the initiation circuit responsible for preparing timely motor program sequences as part of the feedforward control in BG is affected. Consequently, this will affect the timely relay of information to the motor and premotor cortices, such as the IFG and ventral premotor area, for the translation of motor programs to the articulatory commands for production (Chang \& Guenther, 2020). It also points to the role of beta oscillations within this loop that may provide coordination of motor and auditory components for the temporally precise speech sequences. Within the framework of the SFC model, the reduced FA along with increased beta suppression at the mouth motor area may be interpreted as reduced or weak feedforward modeling (Houde \& Nagarajan, 2011). The internal loop within the SFC model includes the feedforward model that captures the sensory predictions of the upcoming motor plan and are encoded in projections from the premotor cortex to auditory and somatosensory cortices. This feedforward modeling is proposed to be captured in the beta component of the fine-grained temporal neural oscillations (Jenson et al., 2014, 2020).

\subsection{Relationship between probabilistic tractography and beta oscillations findings}


The results of the two connectivity directions point to a higher FA from the putamen to the localized MEG peak at the mouth motor area through the thalamus (first direction). The FA in the first connectivity direction was higher in the AWS group compared to the FS group. The connection from the putamen to the cortical areas has been hypothesized to have a role in serial coordination, selection, initiation, inhibition, and timing of actions in motor control (Alario et al., 2006; Jaffard et al., 2008). It should be noted that while probabilistic tractography provides information regarding the likelihood of connections between seed and target voxels, tensor diffusion does not provide rich enough information to pursue multiple pathway propagation options. However, the structural connectivity partly shapes the functional coupling between different neuronal populations (Hagmann et al., 2008), which can be highlighted by correlating the activity of different brain areas using non-invasive neuroimaging such as MEG. The directionality and propagation latencies along large fibers can impact these functional neural dynamics (Petkoski et al., 2016). Our rationale was to use these task-specific functional seed and target masks (based on MEG peak activation) by which the timing and the preferred direction of functional influences may be uncovered. We hypothesized that extracting the diffusion metrices in two opposite directions and investigating the connectivity patterns (Putamen -> MEG peak and MEG peak -> Putamen) based on these functionally-defined masks may point to deficient connections from BG to the primary motor cortex (direction one) as opposed to primary motor cortex to BG (direction two). We acknowledge that this interpretation is not based on direct directionality measures and needs to be confirmed in future studies.

In a study by Lu et al. (2010), the researchers found structural differences in the BGTC loop between AWS and control groups, notably they reported higher gray matter volume concentration in the left putamen and less gray matter volume concentration in the left medial 
frontal gyrus. In addition, basal ganglia activity has been found to be normalized after fluencyshaping therapies (Giraud et al., 2008; Kell et al., 2009). Our current findings along with the previous findings may point, firstly, to an overactivation in the subcortical structure of the basal ganglia, including putamen, which may occur in order to overcome a deficient feedforward control, and secondly, to an under-activation in the cortical motor and premotor areas. In particular, this may affect the ventral premotor area responsible for translating the speech motor programs to articulatory commands for production. This area in the left hemisphere is proposed to be one of the locations for the speech sound map by the DIVA model (Guenther et al, 2006). The structural and functional connectivity deficits may therefore result in an insufficient readout of the motor programs, which in turn causes an impairment in the pathways responsible for feedforward control (Cai et al., 2014). This network deficit of the BGTC loop may cause difficulty in the selection and sequencing of speech movements observed in stuttering. The BGTC loop has also been computationally modeled and impaired connectivity within the basal ganglia-thalamus-ventral premotor cortex in stuttering has been reported (Civier et al., 2013).

The reduction of FA in the left hemisphere compared to the right hemisphere of the AWS group for the first direction (from putamen to cortex) may be interpreted as an insufficient control signal sent by the left hemisphere through feedforward control. These deficits may decrease the automaticity of speech and increase its reliance on auditory feedback. Auditory feedback control is proposed to be more right-lateralized in order to refine speech motor output based on external auditory feedback (Behroozmand et al., 2018; Tourville \& Guenther, 2011; Toyomura et al., 2007). This may cause the right hemisphere to compensate for the incoordination and out-of-sync initiation and/or termination of movements sequences that are normally controlled by the left-lateralized feedforward mechanisms (Tourville \& Guenther, 
2011). However, the right hemisphere is not fully capable of compensating for structural and functional deficits of the left hemisphere found in this work and in previous studies (Beal et al., 2013; Cai et al., 2014; Lu et al., 2010; Mersov et al., 2016). Cai et al. (2014) found a negative correlation of left-lateralized tracts and a positive correlation of right-lateralized tracts with stuttering severity. This also suggests a shift of speech control to the right hemisphere, possibly adopted as a compensatory mechanism and may explain prior findings of hyperactivity in the right hemisphere in stuttering using functional MRI and PET (De Nil \& Kroll, 2001; Ingham et al., 2000; Neef et al., 2018). The resulting shift in the balance of feedforward and feedback control may underlie timing-related disturbances such as initiation, repetition, and termination errors seen in stuttering. The reduction of MD in the right hemisphere would also be noteworthy, but, we did not find any group difference for MD as both groups showed higher MD in the left compared to the right hemisphere in both connectivity directions. Previous findings of probabilistic tractography found increased MD in a sub-region of the left corticospinal tract (CST), and in the bilateral frontal aslant tract (FAT) in AWS compared to fluent speakers (Kronfeld-Duenias et al., 2016). FAT connects the IFG with SMA and preSMA (Catani et al., 2013). It appears that the pattern of reduced FA and increased MD in the left hemisphere is associated with less directional diffusion and thicker than normal fibers in the BGTC loop.

\subsection{Relationship between TBSS and probabilistic tractography results}

The TBSS findings of regional FA reduction in the left ventral premotor area is consistent with the lower FA values extracted from the first connectivity pathway within the BGTC loop in the left compared to the right hemisphere. However, we did not observe any difference in FA in the ventral premotor cortex in the right hemisphere between the two groups using TBSS. This may suggest a connectivity deficit within the BGTC loop that can be better detected using a 
connectivity analysis rather than investigating the white matter deficits underlying one region of interest (i.e., right ventral premotor area).

With regards to MD, using TBSS analysis we found higher MD in parts of the superior thalamic radiations in the right hemisphere in AWS compared to the control group, however, using the connectivity analysis we did not find any group differences of MD for either of the connectivity directions within the BGTC loop. This finding may be suggestive that MD values may not be sufficiently sensitive to show microstructural deficits within this loop, as compared to the FA. As there are not many studies that focused on MD changes within the BGTC loop in stuttering, it would be of interest to investigate this metric in future studies with a larger sample size in adults and also in children who stutter to see how developmental trajectories affect the structural neural underpinning of stuttering behaviour such as structural integrity and myelination. One study looked at the BGTC loop using probabilistic tractography in childhood. Chang and Zhu (2013) investigated structural connectivity in 3- to 9-year-old children with stuttering and found significantly lower than normal probabilistic tract density in the BGTC loop. However, they did not extract the FA or MD values within this loop. Hence, it is difficult to directly relate their finding with the current results as both the population and the analysis pipeline are somewhat different. In another study by Chang et al. (2011) that focused on adults who stutter, they found functional but not structural connectivity differences in the thalamocortical loop of AWS compared to the FS control group. The seed region that they used for structural connectivity was the ventral lateral nucleus (VLN) of the thalamus without the inclusion of the basal ganglia in either of their connectivity analysis. This may explain why the authors did not find similar structural connectivity differences to those found in the present study. 


\subsection{TBSS findings: clusters with no overlap with beta oscillations}

The TBSS findings reported here were mostly consistent with previous findings in the literature showing lower FA and higher MD clusters in AWS compared to the FS group (Cai et al., 2014; Chang et al., 2008; Connally et al., 2013; Neef et al., 2018). Additionally, the reduction in FA in right cingulum and an increase in MD in the left white matter underlying precuneus and posterior cingulate cortex may point to the structural deficits in the cingulum that have been reported previously in the AWS group in one study (Kronfeld-Duenais et al., 2018). KronfeldDuenais suggested that the cingulum may be associated with emotional and interpersonal processing that is affected in AWS. The cingulum is a major limbic pathway that contains long and short association fibers which connects the cingulate cortex with the parahippocampal gyrus, medial prefrontal cortex, and medial cortical regions in the parietal and occipital lobes (Mori \& Aggarwal, 2014). Microstructural properties of the cingulum and precuneus is associated with the ability to infer intentions (Herbet et al., 2014), self-consciousness (Lou et al., 2004), and regulate attention to negative interpersonal stimuli (Keedwell et al., 2016). However, we did not test this hypothesis and these relationships need to be investigated in future investigations.

\subsection{Limitations and future directions}

This study has some limitations that need to be considered when interpreting the results. Firstly, the sample size is relatively small (11 participants in each group), however, this is the first time that combined MEG and structural MRI approach have been applied on the same group of participants, and the results should be generalized with caution. Future studies could benefit from a larger sample size in order to confirm the current findings along with controlling for the type and frequency of stuttering therapy in the AWS group. Secondly, we acknowledge that the TBSS findings from our current study based on uncorrected $p$-value may not be representative of 
the underlying deficits in the AWS group. We mainly carried out this analysis to replicate prior findings reported in the literature. Our main focus was on the results from the probabilistic tractography. In general, TBSS analysis is a voxel-wise method that detects differences in specific regions in standard (template) space, but these regions may map to different tracts in different individuals, due to individual differences in brain anatomy. In contrast, probabilistic tractography approach, is a computational process in which the diffusion data is used to reconstruct continuous $3 \mathrm{D}$ trajectories based on local estimates of fiber orientations. Tract reconstruction is implemented in the native space of each participant, which makes this methodology less prone to inter-subject registration errors, and it is more sensitive than voxelwise approaches like TBSS (Ben-Shachar et al., 2007; Kronfeld-Duenias et al., 2018). In addition, future studies would benefit from including auditory cortical regions such as the superior temporal gyrus within the BGTC loop in stuttering especially as it pertains to the role of auditory feedback control. This will be important in understanding the speech motor network deficits in stuttering and will be informative for refining the theoretical models of speech production.

\subsection{Conclusion}

We associate for the first time, measures of functional beta band oscillations involved in movement preparation and feedforward control with deficient structural integrity in regions including the ventral premotor cortex in the BGTC loop. Our findings indicate reduced FA and increased beta suppression in the left ventral premotor cortex which contains the articulatory commands for speech production and is part of the BGTC loop in AWS. This region-specific functional and structural difference at the cortical level was accompanied by targeted connectivity findings using localized beta band oscillation peaks of the articulatory motor area 
that showed reduced FA and increased MD in the pathways connecting basal ganglia to the cortex through the thalamus in the left hemisphere. Our findings support the notion that stuttering is associated with deficits in structural connectivity between different regions of speech motor network, specifically from the basal ganglia to motor and premotor cortices. These structural network deficits may affect the forward functional flow of information responsible for temporally precise production of speech sequences. 


\section{Acknowledgments}

Funding: this work was supported by research grants from the Natural Sciences and Engineering Research Council of Canada (to LDN) and the Canadian Institutes of Health Research (to DC). The authors also would like to acknowledge Marc Lalancette and Silvia Isabella for their assistance with collecting the brain imaging data and Sonya Bells with data analysis.

\section{Data Statement}

Anonymized datasets recorded and analyzed during this study are available from the corresponding author within the limits of participants consent.

\section{Author Contribution}

Fatemeh Mollaei: Conceptualization, Methodology, Visualization, Formal analysis, Writing Original draft

Anna Mersov: Investigation, Writing - Review and Editing

Merron Woodbury: Software, Writing - Review and Editing

Cecilia Jobst: Software, Investigation, Writing - Review and Editing

Douglas Cheyne: Supervision, Methodology, Writing - Review and Editing, Funding acquisition

Luc De Nil: Supervision, Conceptualization, Writing - Review and Editing, Funding acquisition 


\section{References}

Alm, P. A. (2004). Stuttering and the basal ganglia circuits: A critical review of possible relations. Journal of Communication Disorders, 37(4), 325-369.

Bartolo, R., \& Merchant, H. (2015). B oscillations are linked to the initiation of sensory-cued movement sequences and the internal guidance of regular tapping in the monkey. Journal of Neuroscience, 35(11), 4635-4640.

Basser, P. J., Mattiello, J., \& LeBihan, D. (1994). MR diffusion tensor spectroscopy and imaging. Biophysical Journal, 66(1), 259-267.

Beal, D. Brain development in stuttering (2013). Stuttering Foundation Newsletter. https://www.stutteringhelp.org/brain-development-stuttering.

Beaulieu, C. (2014). The biological basis of diffusion anisotropy. Diffusion MRI (pp. 155-183) Elsevier.

Behrens, T. E., Berg, H. J., Jbabdi, S., Rushworth, M. F., \& Woolrich, M. W. (2007).

Probabilistic diffusion tractography with multiple fibre orientations: What can we gain? NeuroImage, 34(1), 144-155.

Behrens, T. E., Woolrich, M. W., Jenkinson, M., Johansen-Berg, H., Nunes, R. G., Clare, S., . . Smith, S. M. (2003). Characterization and propagation of uncertainty in diffusion-weighted MR imaging. Magnetic Resonance in Medicine: An Official Journal of the International Society for Magnetic Resonance in Medicine, 50(5), 1077-1088.

Behroozmand, R., Phillip, L., Johari, K., Bonilha, L., Rorden, C., Hickok, G., \& Fridriksson, J. (2018). Sensorimotor impairment of speech auditory feedback processing in aphasia. NeuroImage, 165, 102-111.

Ben-Shachar, M., Dougherty, R. F., \& Wandell, B. A. (2007). White matter pathways in reading. Current opinion in neurobiology, 17(2), 258-270.

Cai, S., Tourville, J. A., Beal, D. S., Perkell, J. S., Guenther, F. H., \& Ghosh, S. S. (2014). Diffusion imaging of cerebral white matter in persons who stutter: Evidence for networklevel anomalies. Frontiers in Human Neuroscience, 8, 54.

Catani, M., Howard, R. J., Pajevic, S., \& Jones, D. K. (2002). Virtual in vivo interactive dissection of white matter fasciculi in the human brain. Neuroimage, 17(1), 77-94.

Catani, M., Mesulam, M. M., Jakobsen, E., Malik, F., Martersteck, A., Wieneke, C., .. . Weintraub, S. (2013). A novel frontal pathway underlies verbal fluency in primary progressive aphasia. Brain, 136(8), 2619-2628. 
Chang, S., Erickson, K. I., Ambrose, N. G., Hasegawa-Johnson, M. A., \& Ludlow, C. L. (2008). Brain anatomy differences in childhood stuttering. NeuroImage, 39(3), 1333-1344.

Chang, S., \& Guenther, F. H. (2020). Involvement of the cortico-basal ganglia-thalamocortical loop in developmental stuttering. Frontiers in Psychology, 10

Chang, S. E., Horwitz, B., Ostuni, J., Reynolds, R., \& Ludlow, C. L. (2011). Evidence of left inferior frontal-premotor structural and functional connectivity deficits in adults who stutter. Cerebral Cortex, 21(11), 2507-2518.

Chang, S., \& Zhu, D. C. (2013). Neural network connectivity differences in children who stutter. Brain, 136(12), 3709-3726.

Chang, S., Zhu, D. C., Choo, A. L., \& Angstadt, M. (2015). White matter neuroanatomical differences in young children who stutter. Brain, 138(3), 694-711.

Clinard, C. G., Tremblay, K. L., \& Krishnan, A. R. (2010). Aging alters the perception and physiological representation of frequency: Evidence from human frequency-following response recordings. Hearing Research, 264(1-2), 48-55.

Cohen, J. (1992). A power primer. Psychological Bulletin, 112(1), 155.

Connally, E. L., Ward, D., Howell, P., \& Watkins, K. E. (2014). Disrupted white matter in language and motor tracts in developmental stuttering. Brain and Language, 131, 25-35.

Cox, R. W. (1996). AFNI: Software for analysis and visualization of functional magnetic resonance neuroimages. Computers and Biomedical Research, 29(3), 162-173.

De Nil, L. F., \& Kroll, R. M. (2001). Searching for the neural basis of stuttering treatment outcome: Recent neuroimaging studies. Clinical Linguistics \& Phonetics, 15(1-2), 163-168.

Etchell, A. C., Johnson, B. W., \& Sowman, P. F. (2014). Behavioral and multimodal neuroimaging evidence for a deficit in brain timing networks in stuttering: a hypothesis and theory. Frontiers in human neuroscience, 8, 467.

Etchell, A. C., Johnson, B. W., \& Sowman, P. F. (2015). Beta oscillations, timing, and stuttering. Frontiers in human neuroscience, 8, 1036.

Fischl, B. (2012). FreeSurfer. NeuroImage, 62(2), 774-781.

Fries, P. (2005). A mechanism for cognitive dynamics: Neuronal communication through neuronal coherence. Trends in Cognitive Sciences, 9(10), 474-480.

Fujioka, T., Trainor, L. J., Large, E. W., \& Ross, B. (2012). Internalized timing of isochronous sounds is represented in neuromagnetic beta oscillations. Journal of Neuroscience, 32(5), 1791-1802. 
Gehrig, J., Wibral, M., Arnold, C., \& Kell, C. A. (2012). Setting up the speech production network: How oscillations contribute to lateralized information routing. Frontiers in Psychology, 3, 169.

Giraud, A., Neumann, K., Bachoud-Levi, A., von Gudenberg, A. W., Euler, H. A., Lanfermann, H., \& Preibisch, C. (2008). Severity of dysfluency correlates with basal ganglia activity in persistent developmental stuttering. Brain and Language, 104(2), 190-199.

Guenther, F. H. (2016). Neural control of speech. Cambridge, MA: MIT Press.

Guenther, F. H., Ghosh, S. S., \& Tourville, J. A. (2006). Neural modeling and imaging of the cortical interactions underlying syllable production. Brain and Language, 96(3), 280-301.

Hari, R., Salmelin, R., Mäkelä, J. P., Salenius, S., \& Helle, M. (1997). Magnetoencephalographic cortical rhythms. International Journal of Psychophysiology, 26(1-3), 51-62.

Hagmann, P., Cammoun, L., Gigandet, X., Meuli, R., Honey, C. J., Wedeen, V. J., \& Sporns, O. (2008). Mapping the structural core of human cerebral cortex. PLoS Biol, 6(7), e159.

Herbet, G., Lafargue, G., Bonnetblanc, F., Moritz-Gasser, S., Menjot de Champfleur, N., \& Duffau, H. (2014). Inferring a dual-stream model of mentalizing from associative white matter fibres disconnection. Brain, 137(3), 944-959.

Hickok, G. (2012). Computational neuroanatomy of speech production. Nature Reviews Neuroscience, 13(2), 135-145.

Hickok, G., \& Poeppel, D. (2007). The cortical organization of speech processing. Nature Reviews Neuroscience, 8(5), 393-402.

Houde, J. F., \& Nagarajan, S. S. (2011). Speech production as state feedback control. Frontiers in Human Neuroscience, 5, 82.

Ingham, R. J., Fox, P. T., Ingham, J. C., \& Zamarripa, F. (2000). Is overt stuttered speech a prerequisite for the neural activations associated with chronic developmental stuttering? Brain and Language, 75(2), 163-194.

Jenson, D., Bowers, A. L., Hudock, D., \& Saltuklaroglu, T. (2020). The application of EEG mu rhythm measures to neurophysiological research in stuttering. Frontiers in Human Neuroscience, 13

Jenson, D., Thornton, D., Saltuklaroglu, T., \& Harkrider, A. (2014). Speech perception, production, and the sensorimotor mu rhythm. Paper presented at the Proceedings of the 2014 Biomedical Sciences and Engineering Conference, 1-4.

Jobst, C., Ferrari, P., Isabella, S., \& Cheyne, D. (2018). BrainWave: A matlab toolbox for beamformer source analysis of MEG data. Frontiers in Neuroscience, 12, 587. 
Keedwell, P. A., Doidge, A. N., Meyer, M., Lawrence, N., Lawrence, A. D., \& Jones, D. K. (2016). Subgenual cingulum microstructure supports control of emotional conflict. Cerebral Cortex, 26(6), 2850-2862.

Kell, C. A., Neumann, K., von Kriegstein, K., Posenenske, C., von Gudenberg, A. W., Euler, H., \& Giraud, A. (2009). How the brain repairs stuttering. Brain, 132(10), 2747-2760.

Kikuchi, Y., Okamoto, T., Ogata, K., Hagiwara, K., Umezaki, T., Kenjo, M., . . Tobimatsu, S. (2017). Abnormal auditory synchronization in stuttering: A magnetoencephalographic study. Hearing Research, 344, 82-89.

Kilavik, B. E., Zaepffel, M., Brovelli, A., MacKay, W. A., \& Riehle, A. (2013). The ups and downs of beta oscillations in sensorimotor cortex. Experimental Neurology, 245, 15-26.

Kronfeld-Duenias, V., Amir, O., Ezrati-Vinacour, R., Civier, O., \& Ben-Shachar, M. (2016). The frontal aslant tract underlies speech fluency in persistent developmental stuttering. Brain Structure and Function, 221(1), 365-381.

Kronfeld-Duenias, V., Civier, O., Amir, O., Ezrati-Vinacour, R., \& Ben-Shachar, M. (2018). White matter pathways in persistent developmental stuttering: Lessons from tractography. Journal of Fluency Disorders, 55, 68-83.

Liljeström, M., Kujala, J., Stevenson, C., \& Salmelin, R. (2015). Dynamic reconfiguration of the language network preceding onset of speech in picture naming. Human Brain Mapping, 36(3), 1202-1216.

Lou, H. C., Luber, B., Crupain, M., Keenan, J. P., Nowak, M., Kjaer, T. W., ... \& Lisanby, S. H. (2004). Parietal cortex and representation of the mental self. Proceedings of the National Academy of Sciences, 101(17), 6827-6832.

Lu, C., Peng, D., Chen, C., Ning, N., Ding, G., Li, K., . . Lin, C. (2010). Altered effective connectivity and anomalous anatomy in the basal ganglia-thalamocortical circuit of stuttering speakers. Cortex, 46(1), 49-67.

Max, L., Guenther, F. H., Gracco, V. L., Ghosh, S. S., \& Wallace, M. E. (2004). Unstable or insufficiently activated internal models and feedback-biased motor control as sources of dysfluency: A theoretical model of stuttering. Contemporary Issues in Communication Science and Disorders, 31(Spring), 105-122.

Mersov, A., Cheyne, D., Jobst, C., \& De Nil, L. (2018). A preliminary study on the neural oscillatory characteristics of motor preparation prior to dysfluent and fluent utterances in adults who stutter. Journal of Fluency Disorders, 55, 145-155.

Mersov, A., Jobst, C., Cheyne, D. O., \& De Nil, L. (2016). Sensorimotor oscillations prior to speech onset reflect altered motor networks in adults who stutter. Frontiers in Human Neuroscience, 10, 443. 
Mori, S., \& Aggarwal, M. (2014). In vivo magnetic resonance imaging of the human limbic white matter. Frontiers in aging neuroscience, 6, 321.

Nambu, A. (2011). Somatotopic organization of the primate basal ganglia. Frontiers in Neuroanatomy, 5, 26.

Neef, N. E., Anwander, A., Bütfering, C., Schmidt-Samoa, C., Friederici, A. D., Paulus, W., \& Sommer, M. (2018). Structural connectivity of right frontal hyperactive areas scales with stuttering severity. Brain, 141(1), 191-204.

Oldfield, R. C. (1971). The assessment and analysis of handedness: The edinburgh inventory. Neuropsychologia, 9(1), 97-113.

Organisation mondiale de la santé, World Health Organization, \& World Health Organization Staff. (2001). International classification of functioning, disability and health: ICF. World Health Organization.

Petkoski, S., Spiegler, A., Proix, T., Aram, P., Temprado, J. J., \& Jirsa, V. K. (2016). Heterogeneity of time delays determines synchronization of coupled oscillators. Physical Review E, 94(1), 012209.

Pfurtscheller, G. (1981). Central beta rhythm during sensorimotor activities in man. Electroencephalography and Clinical Neurophysiology, 51(3), 253-264.

Piai, V., Roelofs, A., Rommers, J., \& Maris, E. (2015). Beta oscillations reflect memory and motor aspects of spoken word production. Human Brain Mapping, 36(7), 2767-2780.

Pierpaoli, C., \& Basser, P. J. (1996). Toward a quantitative assessment of diffusion anisotropy. Magnetic Resonance in Medicine, 36(6), 893-906.

Rech, F., Wassermann, D., \& Duffau, H. (2020). New insights into the neural foundations mediating movement/language interactions gained from intrasurgical direct electrostimulations. Brain and Cognition, 142, 105583.

Reilly, S., Onslow, M., Packman, A., Wake, M., Bavin, E. L., Prior, M., . . Ukoumunne, O. C. (2009). Predicting stuttering onset by the age of 3 years: A prospective, community cohort study. Pediatrics, 123(1), 270-277.

Riley, G. D. (1972). A stuttering severity instrument for children and adults. Journal of Speech and Hearing Disorders, 37(3), 314-322.

Robinson, S. E., \& Vrba, J. (1999). Functional neuroimaging by synthetic aperture magnetometry (SAM). In T. Yoshimoto et al., (Eds.), Recent Advances in Biomagnetism (pp. 302-305). Tohoku University Press. 
Salmelin, R., \& Sams, M. (2002). Motor cortex involvement during verbal versus non-verbal lip and tongue movements. Human Brain Mapping, 16(2), 81-91.

Saltuklaroglu, T., Harkrider, A. W., Thornton, D., Jenson, D., \& Kittilstved, T. (2017). EEG mu $(\mu)$ rhythm spectra and oscillatory activity differentiate stuttering from non-stuttering adults. NeuroImage, 153, 232-245.

Schnitzler, A., Gross, J., \& Timmermann, L. (2000). Synchronised oscillations of the human sensorimotor cortex. Acta Neurobiologiae Experimentalis, 60(2), 271-288.

Sengupta, R., Shah, S., Gore, K., Loucks, T., \& Nasir, S. M. (2016). Anomaly in neural phase coherence accompanies reduced sensorimotor integration in adults who stutter. Neuropsychologia, 93, 242-250.

Smith, S. M., Jenkinson, M., Johansen-Berg, H., Rueckert, D., Nichols, T. E., Mackay, C. E., . . . Matthews, P. M. (2006). Tract-based spatial statistics: Voxelwise analysis of multi-subject diffusion data. NeuroImage, 31(4), 1487-1505.

Smith, S. M., Jenkinson, M., Woolrich, M. W., Beckmann, C. F., Behrens, T. E., Johansen-Berg, H., ... Flitney, D. E. (2004). Advances in functional and structural MR image analysis and implementation as FSL. NeuroImage, 23, S208-S219.

Smith, S. M., \& Nichols, T. E. (2009). Threshold-free cluster enhancement: Addressing problems of smoothing, threshold dependence and localisation in cluster inference. NeuroImage, 44(1), 83-98.

Sommer, M., Koch, M. A., Paulus, W., Weiller, C., \& Büchel, C. (2002). Disconnection of speech-relevant brain areas in persistent developmental stuttering. The Lancet, 360(9330), 380-383.

Sowman, P. F., Ryan, M., Johnson, B. W., Savage, G., Crain, S., Harrison, E., ... \& Burianová, H. (2017). Grey matter volume differences in the left caudate nucleus of people who stutter. Brain and language, 164, 9-15.

Tourville, J. A., \& Guenther, F. H. (2011). The DIVA model: A neural theory of speech acquisition and production. Language and cognitive processes, 26(7), 952-981.

Toyomura, A., Fujii, T., \& Kuriki, S. (2011). Effect of external auditory pacing on the neural activity of stuttering speakers. NeuroImage, 57(4), 1507-1516.

Toyomura, A., Koyama, S., Miyamaoto, T., Terao, A., Omori, T., Murohashi, H., \& Kuriki, S. (2007). Neural correlates of auditory feedback control in human. Neuroscience, 146(2), 499503.

Watkins, K. E., Smith, S. M., Davis, S., \& Howell, P. (2008). Structural and functional abnormalities of the motor system in developmental stuttering. Brain, 131(1), 50-59. 
World Health Organization (WHO). (2016). International Classification of Functioning, Disability and Health (ICF). Geneva: Switzerland.

Witte, R. S., \& Witte, J. S. (2010). Statistics. Hoboken, NJ: Wiley. 


\section{Figure legends}

Figure 1. MEG beta suppression peak (AWS vs. FS) overlaid on cluster 1 of TBSS FA results (in orange) showing white matter (WM) underlying the ventral premotor cortex where AWS have lower FA compared to FS $(p<0.005$, \# of voxels $=12)$.

Figure 2. Regions of interests (ROIs) and the BGTC circuit: (a) MEG-defined ventral premotor cortex, BA 6 (blue), (b) and anatomically-defined ROIs lower medial putamen (green) and thalamus (blue) overlaid on a representation of the FA maps in the MNI standard space for a participant with developmental stuttering in the left hemisphere, (c) The reconstructed binary images of $1 \%$ of probabilistic maps for the first connectivity direction in FS (left side) and AWS (right side) overlaid on the MNI 152 2mm template (coronal view).

Figure 3. Fractional Anisotropy (FA), and Mean Diffusivity (MD) for the Basal GangliaThalamocortical (BGTC) circuit in two connectivity directions. Direction one: lower medial putamen (seed mask) to MEG-defined cortical ROI (target mask) through thalamus (waypoint mask); Direction two: MEG-defined cortical ROI (seed mask) to lower medial putamen (target mask) for the two groups (FS: fluent speakers, AWS: adults who stutter) and the two hemispheres (blue: left hemisphere, red: right hemisphere). Boxplots provide $25^{\text {th }}$ and $75^{\text {th }}$ percentiles and median. Outliers are indicated by (o). Only significant results that survived the Bonferroni-adjusted $p$ value for multiple comparisons are shown with an $(*<0.05)$. 


\section{Figures}

Figure 1.

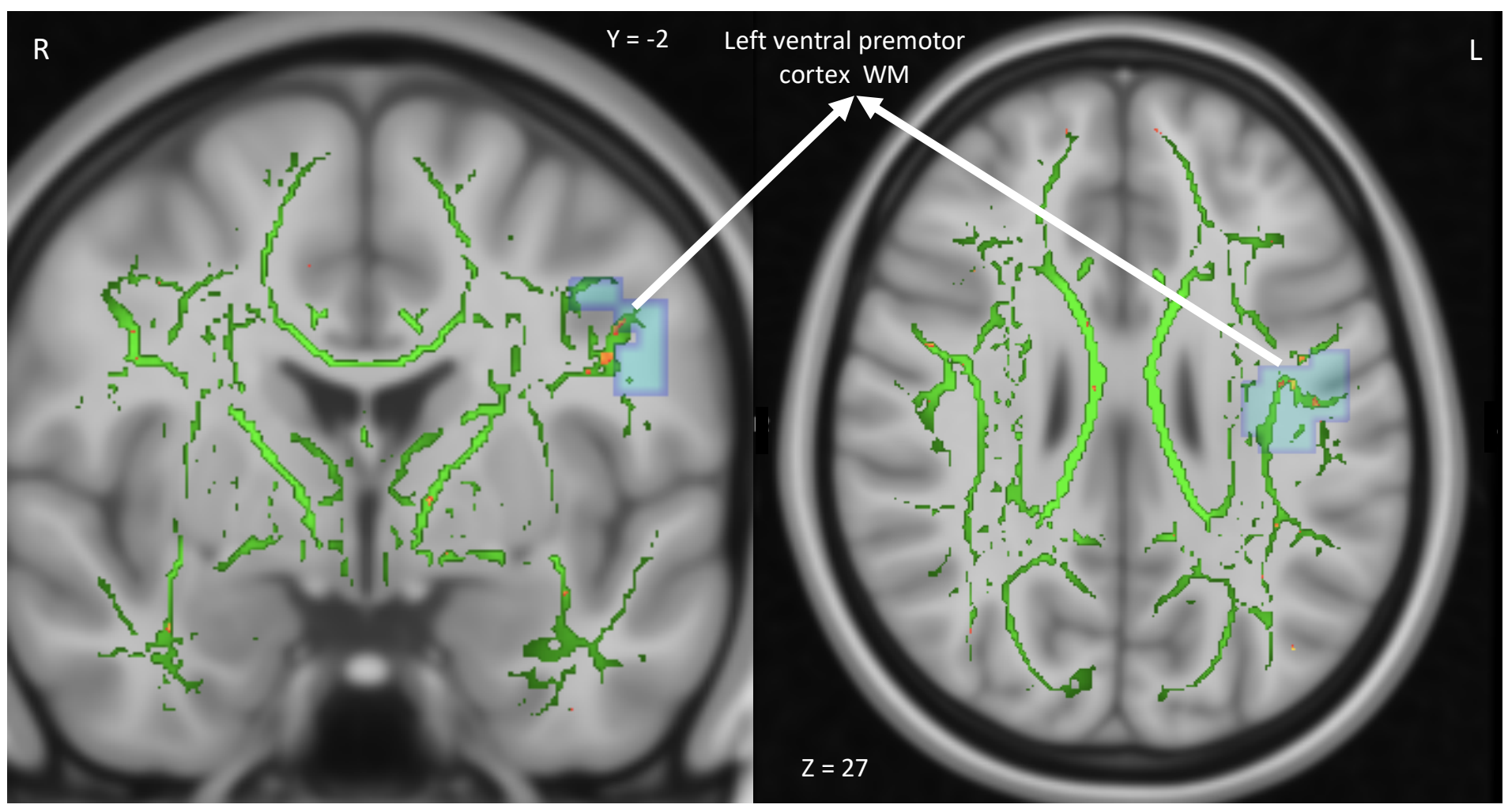


Figure 2.

(a) MEG Peak in the left hemisphere
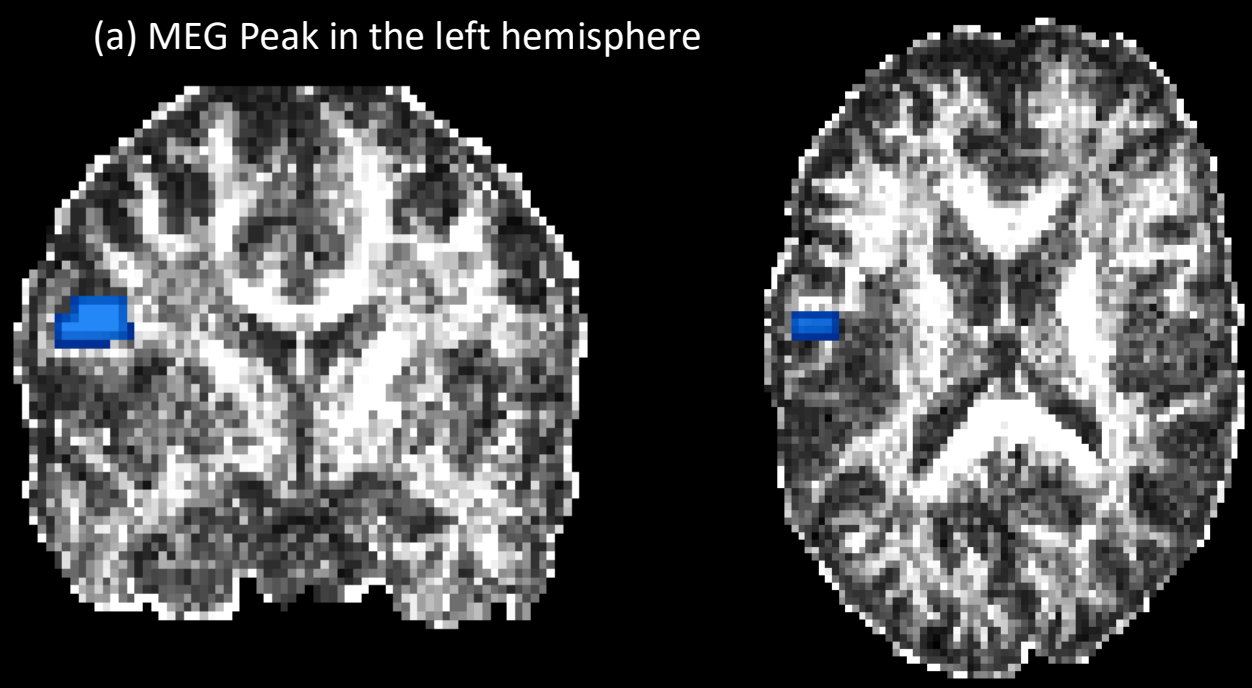

(b) Left Lower Medial Putamen and Thalamus
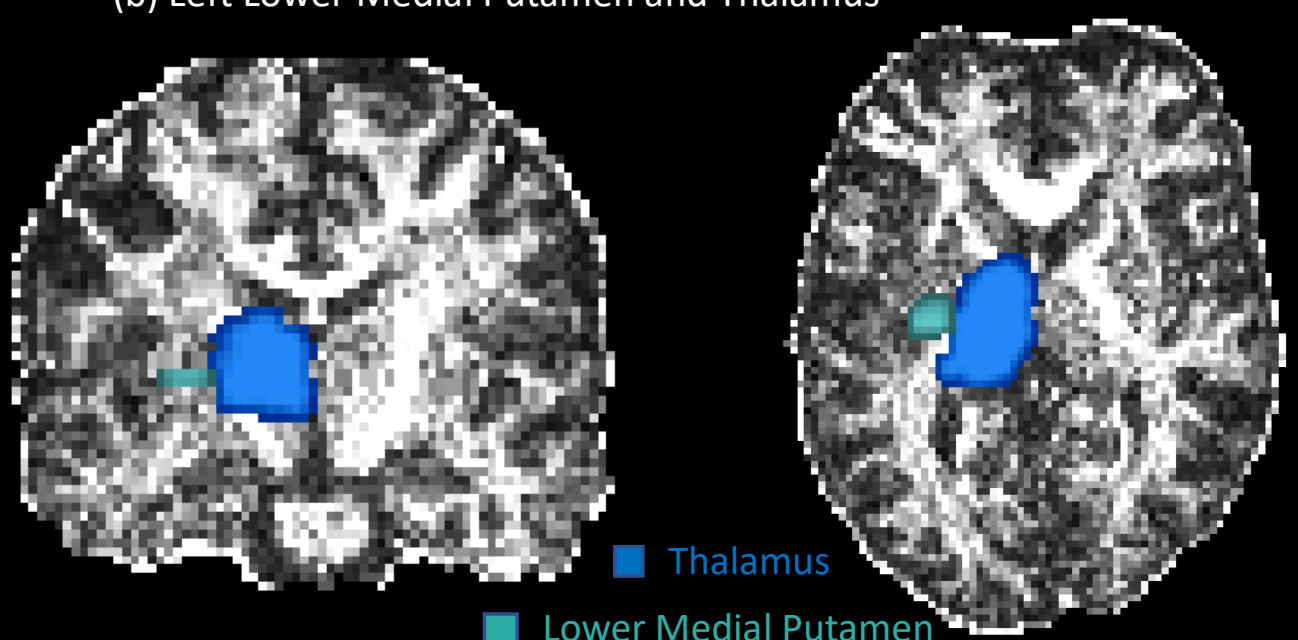

$\square$ Lower Medial Putamen

(c) Reconstructed BGTC circuit
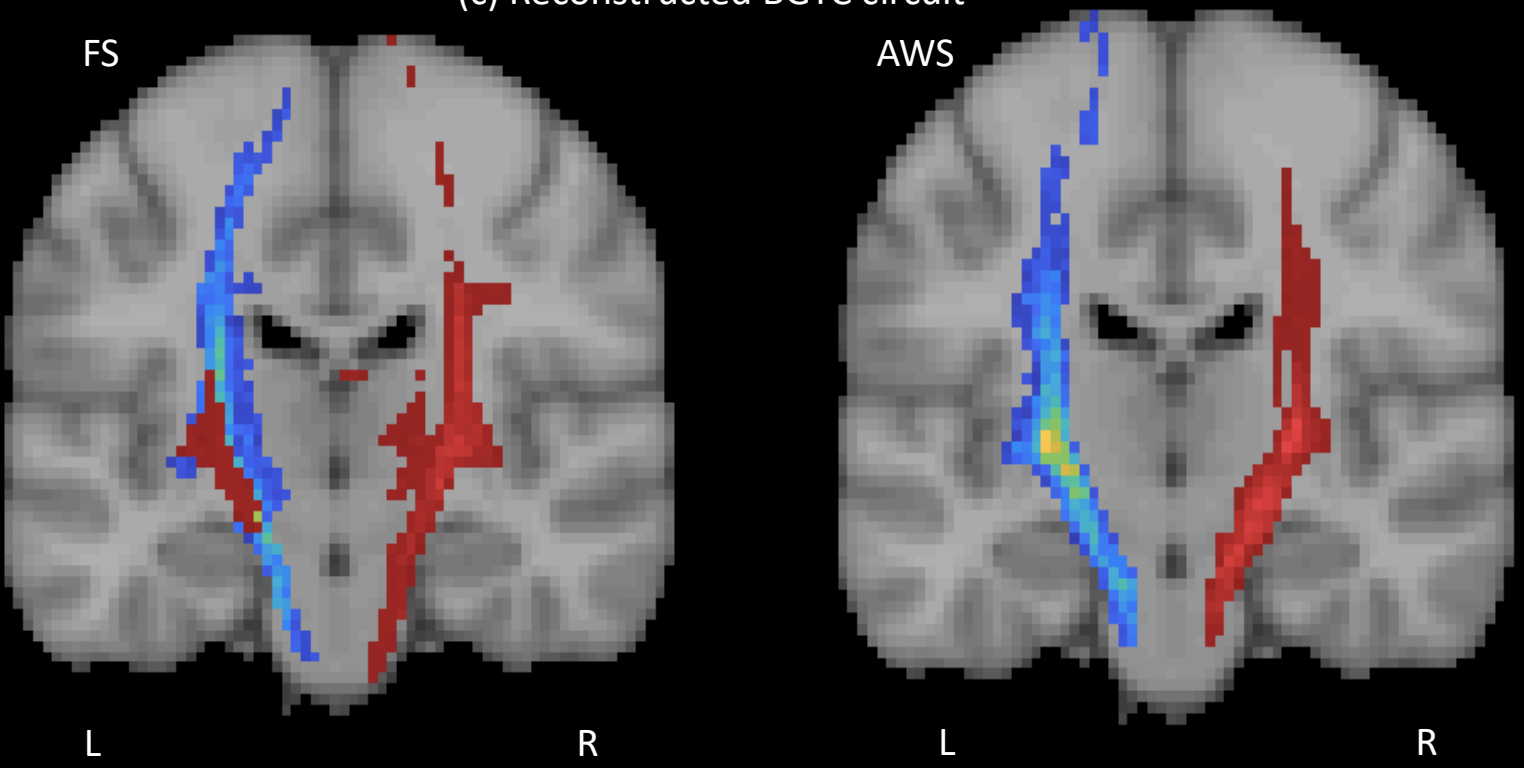
Figure 3.
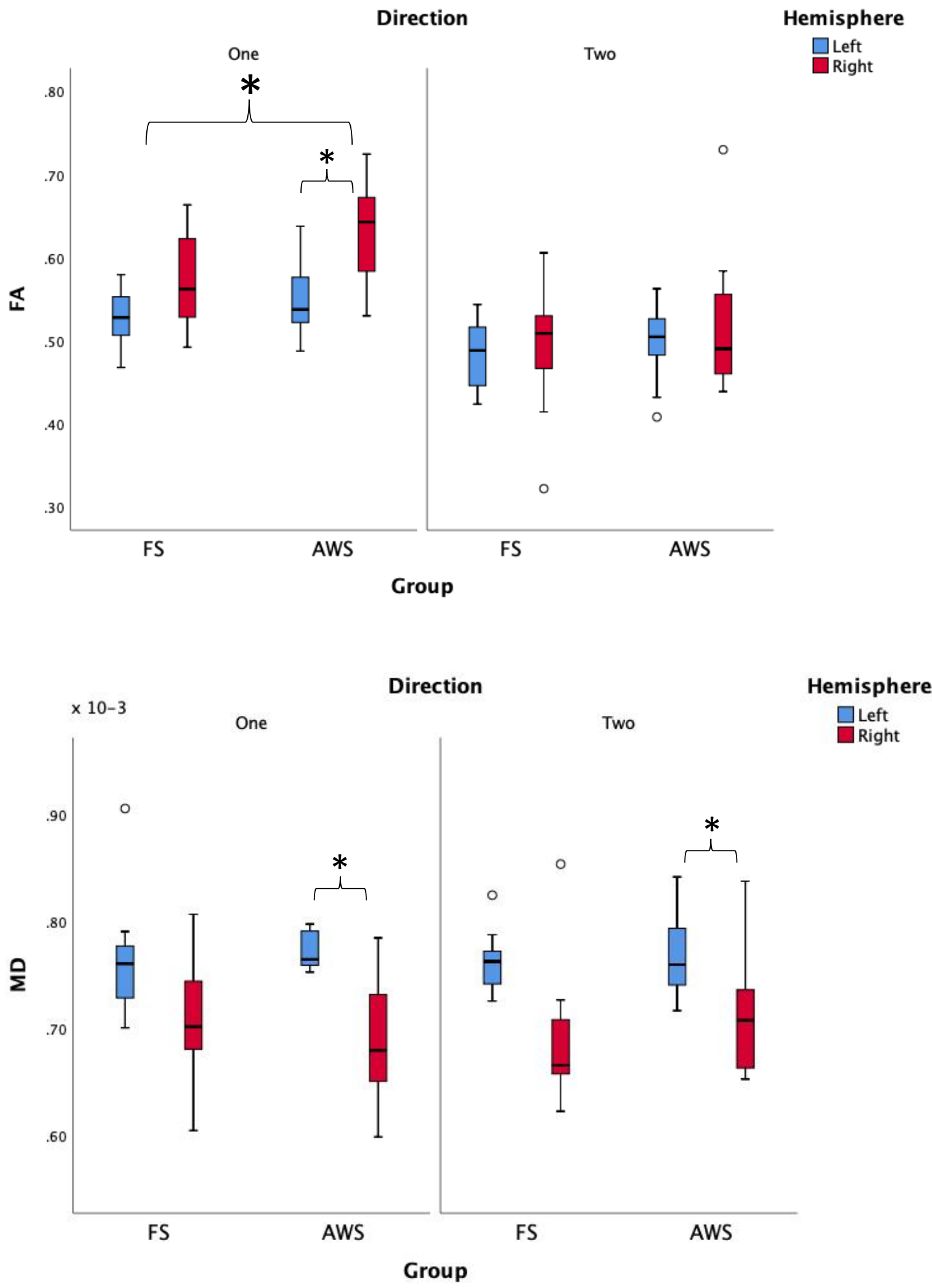


\section{Tables}

Table 1.

Stuttering Severity Scores (SSI-4)

\begin{tabular}{ccc} 
AWS ID & SSI Class & SSI Total \\
\hline \hline S01 & Very mild & 17 \\
S02 & Very mild & 12 \\
S03 & Mild & 18 \\
S04 & Mild & 24 \\
S05 & Mild & 22 \\
S06 & Severe & 32 \\
S07 & Very mild & 16 \\
S08 & Mild & 23 \\
S09 & Very severe & 37 \\
S10 & Mild & 19 \\
S11 & Severe & 34
\end{tabular}

Table 2.

TBSS results: clusters of voxels with significant between-group differences in fractional anisotropy (FA)

\begin{tabular}{|c|c|c|c|c|c|}
\hline $\begin{array}{l}\text { Cluster } \\
\text { number }\end{array}$ & Cluster location & $\begin{array}{c}\text { MNI } \\
\text { coordinates }\end{array}$ & $\begin{array}{l}\text { Number of } \\
\text { voxels }\end{array}$ & $t$-value & Cohen's $d$ \\
\hline & \multicolumn{5}{|l|}{ AWS $<$ FS } \\
\hline 1 & $\begin{array}{l}\text { L superior longitudinal } \\
\text { fasciculus underlying ventral } \\
\text { premotor cortex }\end{array}$ & $(-45,-2,27)$ & 12 & 3.37 & 1.507 \\
\hline 2 & L posterior corona radiata & $(-21,-49,36)$ & 11 & 2.95 & 1.319 \\
\hline 3 & $\mathrm{R}$ anterior corona radiata & $(23,19,16)$ & 11 & 3.58 & 1.601 \\
\hline 4 & $\begin{array}{l}\mathrm{R} \text { superior longitudinal } \\
\text { fasciculus underlying posterior } \\
\text { division of supramarginal gyrus }\end{array}$ & $(53,-37,35)$ & 11 & 3.74 & 1.672 \\
\hline 5 & $\begin{array}{l}\mathrm{L} \text { inferior division of lateral } \\
\text { occipital cortex }\end{array}$ & $(-41,-67,-10)$ & 10 & 3.35 & 1.498 \\
\hline 6 & $\begin{array}{l}\text { R cingulum (hippocampus) } \\
\text { underlying posterior division of } \\
\text { Cingular gyrus }\end{array}$ & $(19,-36,-5)$ & 10 & 3.00 & 1.341 \\
\hline
\end{tabular}


Table 3.

TBSS results: clusters of voxels with significant between-group differences in mean diffusivity (MD)

\begin{tabular}{|c|l|c|c|c|c|}
\hline $\begin{array}{c}\text { Cluster } \\
\text { number }\end{array}$ & \multicolumn{1}{|c}{ Cluster location } & $\begin{array}{c}\text { MNI } \\
\text { coordinates }\end{array}$ & $\begin{array}{c}\text { Number of } \\
\text { voxels }\end{array}$ & \begin{tabular}{c}
$\boldsymbol{t}$-value \\
\hline $\mathbf{1}$
\end{tabular} & $\begin{array}{c}\text { Cohen' } \\
\text { s } \boldsymbol{d}\end{array}$ \\
\hline & $\begin{array}{l}\text { LWS }>\text { FS } \\
\text { L white matter underlying } \\
\text { precuneus and posterior cingulate } \\
\text { cortex }\end{array}$ & $(-1,-60,11)$ & 20 & 3.50 & 1.565 \\
\hline $\mathbf{2}$ & $\begin{array}{l}\text { R superior longitudinal fasciculus } \\
\text { underlying insular cortex }\end{array}$ & $(37,-19,33)$ & 11 & 3.20 & 1.431 \\
\hline $\mathbf{3}$ & $\begin{array}{l}\text { R posterior thalamic radiations } \\
\text { (include optic radiation) }\end{array}$ & $(35,-58,5)$ & 10 & 3.21 & 1.435 \\
\hline $\mathbf{4}$ & $\begin{array}{l}\text { R white matter underlying posterior } \\
\text { division of supramarginal gyrus }\end{array}$ & $(45,-39,44)$ & 10 & 3.04 & 1.359 \\
\hline
\end{tabular}




\section{Supplementary figures}

Figure 1: Clusters showing significant between-group difference in FA values. All the six clusters in this figure contain differences in the direction of AWS $<$ FS.

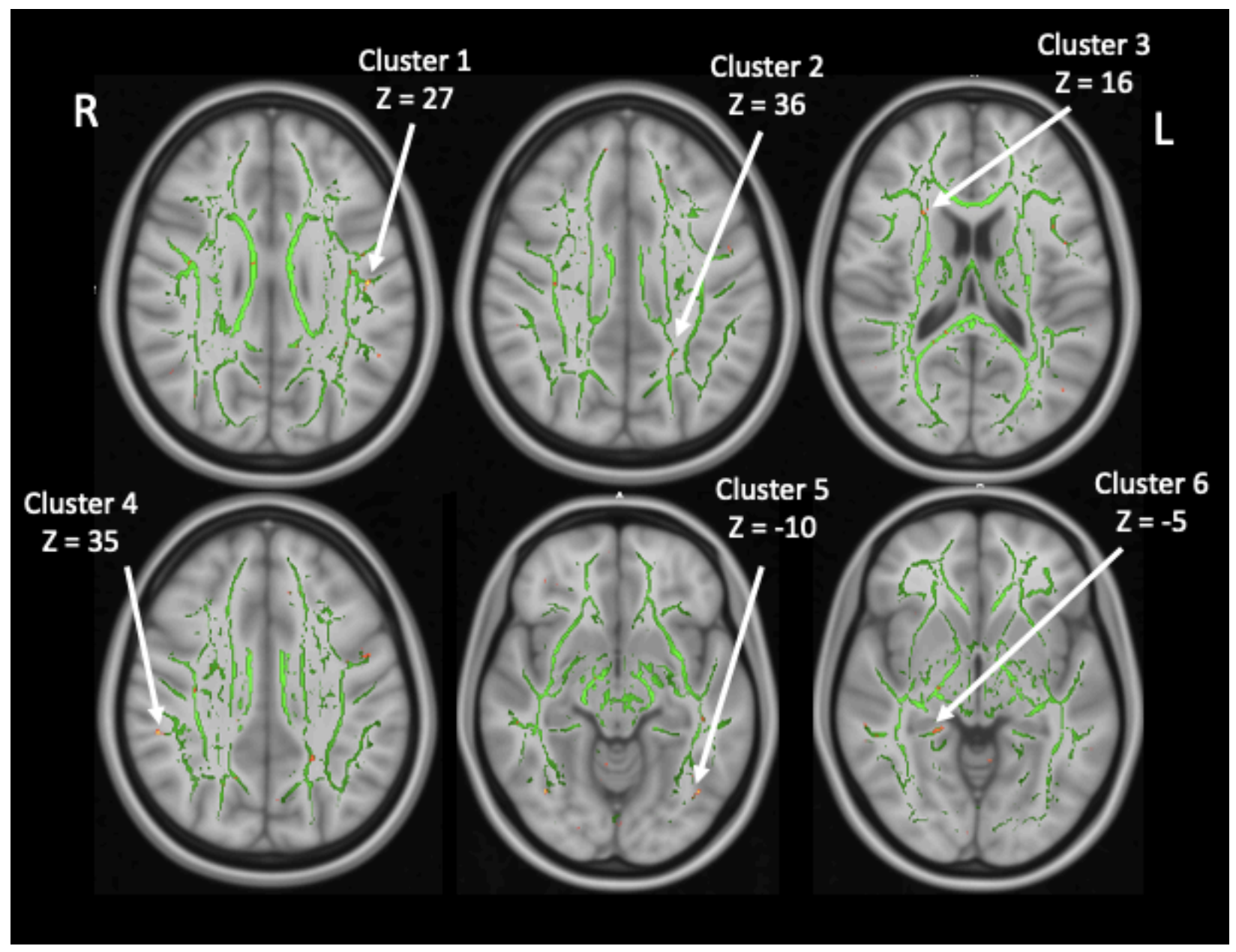


Figure 2: Clusters showing significant between-group difference in MD values. All the four clusters in this figure contain differences in the direction of AWS $>$ FS.

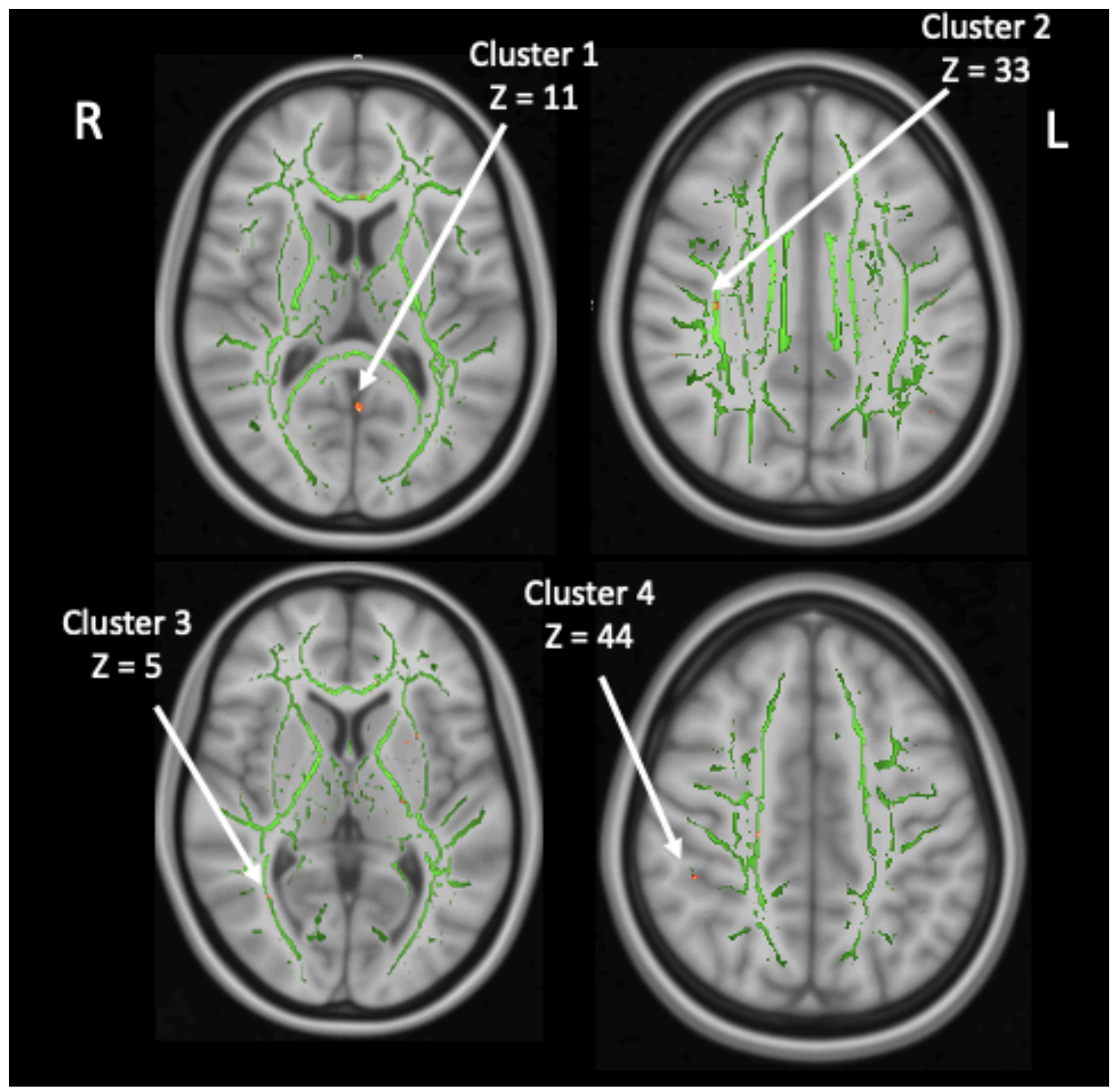

